\title{
The lytic mechanism of Escherichia coli $\alpha$-hemolysin associated to outer membrane vesicles
}

\section{- Lytic action mechanism of OMVs-associated HlyA}

\author{
Vanesa Herlax ${ }^{1 *}$, Maria Florencia Henning ${ }^{1}$, Ana María Bernasconi ${ }^{1}$, Felix María Goñi ${ }^{3}$, Laura Bakás ${ }^{1,2}$ \\ ${ }^{1}$ Instituto de Investigaciones Bioquímicas La Plata (INIBIOLP), CCT-La Plata, CONICET, UNLP, Facultad de Ciencias Médicas, \\ La Plata, Argentina; *Corresponding Author: vherlax@atlas.med.unlp.edu.ar \\ ${ }^{2}$ Departamento de Ciencias Biológicas, Facultad de Ciencias Exactas, Universidad Nacional de La Plata, La Plata, Argentina \\ ${ }^{3}$ Unidad de Biofísica (Centro Mixto CSIC-UPV/EHU), and Departamento de Bioquímica, Universidad del País Vasco, Bilbao, Spain
}

Received 2 January 2010; revised 1 February 2010; accepted 2 February 2010.

\begin{abstract}
Alpha-hemolysin (HlyA) is an extracellular toxin secreted by Escherichia coli, targeting to plasma membranes of eukaryotic cells. Recently it was found that this toxin is released to external media associated to bacterial outer membrane vesicles (OMVs), but the hemolytic mechanism in this way has not been studied yet. Our results report that HlyA is the only protein present in OMVs that is responsible for erythrocyte lysis, and show that no fusion event is involved in the lytic mechanism of OMVs-HlyA. Furthermore, the specific hemolytic activity is approximately 10 fold higher than that of purified free-HlyA, showing the same relative lysis efficiency and specificity for erythrocytes from different species. OMVs could be an important auxiliary way of secretion, acting mainly as a concentration mechanism of HlyA near the target cells. Cell lysis would occur after toxin transfer from OMVs to target membranes, as demonstrated by hemolysis kinetic studies, lipid mixing and western blot assays.
\end{abstract}

Keywords: OMVs; Toxin; Lipid Mixing; Hemolytic Activity; Erythrocytes

\section{INTRODUCTION}

The hemolytic toxin $\alpha$-hemolysin (HlyA), member of the RTX toxin family [1], is an important virulence factor produced by several strains of Escherichia coli. It is involved in human extraintestinal diseases, such as urinary tract infections, peritonitis, meningitis and septicemia [2]. As in most RTX proteins, an operon composed of 4 genes $(h l y A B C D)$ is involved in the polypeptide synthesis, postraslational modification and secretion of the active HlyA to extracellular media [3,4]. HlyA is synthesized in an inactive form (ProHlyA), which is activated in the cytoplasm to the hemolytically active form by $\mathrm{HlyC}$, a fatty acid acyltransferase [5]. Then HlyA is secreted across both membranes by the type I export process employing an uncleaved C-terminal recognition signal $[6,7]$. Although HlyA has its own machinery to be export from the bacteria, Balsalobre et al. [8] demonstrated the presence of physiologically active HlyA in outer membrane vesicles (OMVs) of hemolytic strains of Escherichia coli.

Outer membrane vesicles (OMVs) are constantly being discharged from the surface of Gram negative bacteria during bacterial growth. All Gram negative bacteria studied up to date, including Escherichia coli, Neisseria meningitidis, Pseudomonas aeruginosa, Helicobacter pylori, Shigella flexneri and Actinobacillus actinomycetemcomitans produce outer membrane vesicles and their release is increased when bacteria are exposed to stressful conditions such as antibiotics or serum. One can be tempted to think that OMVs production is the result of membrane instability, but recent studies have demonstrated that mutations that affect protein synthesis, localization, envelope structure and envelope stress response pathway alter the vesiculation levels. In this way the release of OMVs offers to the cell an effective mechanism for removal of material as a macromolecular complex, allowing to discard unwanted material or alter the composition of the envelope under conditions where remodeling would be advantageous $[9,10]$.

Despite that in vivo, release of OMVs could not be monitored, the presence of particles resembling these vesicles has been detected in plasma from patients with different infectious processes [11-18]. OMVs serve as secretory vehicles for proteins and lipids of Gram negative bacteria and play roles in establishing a colon- 
ization niche carrying or transmitting virulence factors into host cells or modulating host defense and response, acting also as long-range virulence factors that can protect luminal cargo from extracellular host proteases and penetrate into tissues more readily than larger bacteria [19]. An extensive review about the significance of bacterial OMVs in the host-pathogen interaction was published by Kuehn and Kesty [20]. Besides the toxin o protein delivery, other roles were characterized for OMVs, such as: interaction interspecies during multispecies infections, DNA transfer, DNA uptake, communication interspcies [21].

OMVs are primarily composed of lipopolysaccharide (LPS), phospholipids and various outer membrane (OM) proteins and periplasmic components, but they do not contain intra membrane (IM) or cytoplasmic components [22], although OMVs isolated from E. coli O157:H7 contain DNA [23].

Considering that HlyA was found associated to OMVs of some strain of E. coli and that erythrocytes have been the cell type classically used in studies on the action mechanism of this toxin, in this research we studied the hemolytic mechanism of OMVs-associated HlyA. The results demonstrate that the lytic action of OMVs-associated HlyA like free-HlyA does not involve fusion events, instead the toxin is transferred from OMVs to target cells, as demonstrated by hemolysis kinetic studies, lipid mixing and western blot assays.

\section{EXPERIMENTAL PROCEDURES}

\subsection{Isolation of Outer Membrane Vesicles OMVs}

E. coli strains used as a source of HlyA were WAM 1824, an overproducing hemolysin strain [24], and WAM 783, for the unacylated inactive toxin ProHlyA [25]. Both of them were kindly provided by $\mathrm{R}$. A. Welch (University of Wisconsin, Madison, Wisconsin).

OMVs were isolated from bacterial culture supernatants. Briefly, bacteria were grown at $37^{\circ} \mathrm{C}$ in Luria Bertani broth (LB). Culture samples from the decelerating growth phase were centrifuged at $10000 \mathrm{rpm}$ for 20 $\min$ at $4^{\circ} \mathrm{C}$. The resulting suspension was then centrifuged at $47500 \mathrm{rpm}$ for $2 \mathrm{~h}$ at $4^{\circ} \mathrm{C}$ to collect the pellet containing OMVs.

\subsection{Protein Purification}

Free-HlyA was purified from the supernatants resulting from OMVs. It was concentrated and partially purified by precipitation with $20 \%$ cold ethanol. A precipitate containing HlyA was collected by centrifugation $(1 \mathrm{~h}$, $10000 \mathrm{rpm}$ in a Sorvall rotor SSA 34), then it was resuspended in $20 \mathrm{mM}$ Tris, $150 \mathrm{mM} \mathrm{NaCl}$, pH 7.0 (TC buffer). This preparation showed, on SDS-PAGE, a main band at $110 \mathrm{kDa}$ corresponding to more than $90 \%$ of the total protein. This band was assigned to free-HlyA. The protein could be stored at $-70^{\circ} \mathrm{C}$.

\subsection{OMVs Composition}

\subsubsection{Quantitative Assays}

Quantitative assays were performed using Lowry method for proteins [26], KDO 2-keto-3-deoxyoctonate for LPS [27] and inorganic phosphorous for phospholipids [28].

\subsubsection{Analysis of Proteins by SDS-PAGE}

Samples were electrophoresed on 10\% acrylamide gels in the presence of SDS according to Laemmli et al. [29]. Protein bands were visualized by Coomassie Blue staining [30].

\subsubsection{Immunoblotting Analysis}

Samples from 10\% SDS-polyacrylamide gels were transferred to nitrocellulose by the method of Towbin et al. [31]. Blots were blocked with 3\% skim milk in TBS buffer (10 mM Tris-HCl, $150 \mathrm{mM} \mathrm{NaCl}, \mathrm{pH} 7.5)$ at room temperature for $2 \mathrm{~h}$. They were then incubated with a solution containing a polyclonal rabbit antihemolysin antibody $(1: 1000)$ in $3 \%$ skim milk-TBS at $4^{\circ} \mathrm{C}$ overnight, washed with TBS buffer, and finally reacted with peroxidase-conjugated anti-rabbit Ig antibody (Sigma) (1:1000) in TBS buffer with 3\% skim milk at room temperature for $2 \mathrm{~h}$. After incubation and washing as stated above, the nitrocellulose was transferred to a peroxidase substrate solution containing 6 $\mathrm{mg}$ 4-chloro-1-naphthol (Sigma) in $1 \mathrm{ml}$ methanol, 1 $\mathrm{ml} \mathrm{TBS,} 3 \mathrm{ml} \mathrm{H}_{2} \mathrm{O}$ and $8 \mu \mathrm{H}_{2} \mathrm{O}_{2}$ for the detection of horseradish peroxidase-conjugate antibodies on the membrane.

\subsection{Hemolytic Assays}

For the hemolytic assays, free-HlyA or OMVs associated-HlyA were serially diluted in TC cold buffer containing $10 \mathrm{mM} \mathrm{CaCl}_{2}$ on a 96 -well microtiter plate. One hundred $\mu \mathrm{L}$ of the diluted suspensions were mixed with $100 \mu \mathrm{l}$ of standardized horse or rabbit erythrocytes. The mixture was incubated at $37^{\circ} \mathrm{C}$ for $30 \mathrm{~min}$. The absorbance of supernatants was read at $412 \mathrm{nM}$ [32]. One hemolytic unit/mL $(\mathrm{HU} / \mathrm{mL})$ is defined as 10 fold the dilution of toxin preparation producing $50 \%$ lysis of the erythrocyte suspension.

The standardization of erythrocytes was done just before the assay. The erythrocytes were washed in $0.9 \%$ $\mathrm{NaCl}$ and then diluted to $12.5 \mu \mathrm{L}$ in $1 \mathrm{~mL}$ of distilled water to give a reading of 0.6 absorbance unit at $412 \mathrm{nM}$ [33]. 


\subsection{Hemolysis Kinetics}

Hemolysis kinetics was determined by measuring the decrease in turbidity (absorbance at $650 \mathrm{nM}$ ) of a standardized horse erythrocyte suspension exposed to freeHlyA or OMVs associated-HlyA as a function of time at $37^{\circ} \mathrm{C}$. A series of hemolytic reactions were set up starting with an amount of free-HlyA or OMVs associated-HlyA that would produce $100 \%$ of hemolysis in 96 -well plate dilution assays. The real time kinetics assays for hemolysis were performed in the following manner: $500 \mu \mathrm{L}$ of standardized erythrocytes and $500 \mu \mathrm{L}$ of hemolysis buffer containing $10 \mathrm{mM} \mathrm{CaCl}$ were placed into a cuvette. Hemolysis started when the toxin was injected into the cuvette, and the absorbance at $650 \mathrm{nM}$ was measured.

\subsection{LUVs Preparation}

Large unilamellar vesicles (LUVs) composed of PC/PE/Chol 2:1:1 molar ratio were prepared by extrusion using 0.1 $\mu \mathrm{M}$ pore size membranes as described by Mayer et al. [34].

\subsection{Ghost Erythrocytes Preparation}

Five mililitres of horse erythrocytes were washed with TC buffer and osmotically lysed in $10 \mathrm{mM}$ tris- $\mathrm{HCl} \mathrm{PH}=7.4$ buffer at $4^{\circ} \mathrm{C}$ for $30 \mathrm{~min}$. The membranes were pelleted by centrifugation (10 min at $10000 \mathrm{rpm})$, and washed until the supernatant remained clear. The membranes were finally resuspended in $3 \mathrm{~mL}$ of TC buffer. The phosphate concentration of these samples was $0.53 \pm 0.05 \mathrm{mg}$ phospholipid/mL $(\mathrm{n}=3)$.

\subsection{Labeling of Ghost Erythrocytes with Rhodamine-Phosphatidylethalonamine (Rh-Pe) and N-(7-Nitro-2, 1, 3-Benzoxa- diazol-4-YI)-Phosphatidylethanolamine (NBD-PE)}

Ghost erythrocytes were labeled with Rh-PE and NBD-PE (Molecular Probes, Eugene, O.R.). Both fluorophores are coupled to the free amino group of phosphatidylethanolamine to provide analogues which can be incurporated into a lipid bilayer. $20 \mu \mathrm{L}$ of a fresh solution of each probe $(1 \mathrm{mg} / \mathrm{ml}$ in ethanol) was added to $75 \mu \mathrm{L}$ of ghost erythrocytes. After incubating the suspension for $1 \mathrm{hr}$ at room temperature, 6 washes with TC were performed so as to separate unbound labeled lipids. Finally the labeled ghost erythrocytes were resuspended in $5.4 \mathrm{ml}$ of TC.

\subsection{Lipid Mixing}

Membrane fusion was analyzed by lipid mixing between OMVs and ghost erythrocytes labeled with NBD-PE and $\mathrm{Rh}-\mathrm{PE}$. The fusion assay involves resonance energy transfer between NBD as donor and Rh as acceptor.
When both fluorescent lipids are in ghost erythrocytes, efficient energy transfer is observed [35]. After lipid mixing by fusion with a population of unlabeled membranes, in this case OMVs, a decrease in efficiency of resonance energy transfer should be observed followed by an increase of the donor fluorescence or decrease of the acceptor fluorescence. In our experimental design, we followed the increase of donor (NBD-PE) fluorescence as a function of time using the excitation and emission monochromators set at $465 \mathrm{nM}$ and $530 \mathrm{nM}$, respectively. Slits for excitation and emission were $4 \mathrm{nM}$.

The extent of lipid mixing was determined according to the following equation:

$$
\% \text { lipid mixing }=\left[\left(\mathrm{F}_{\mathrm{t}}-\mathrm{F}_{0}\right) /\left(\mathrm{F}_{\max }-\mathrm{F}_{0}\right)\right] \times 100
$$

where $F_{0}$ is the initial fluorescence value of erythrocytes labeled with both probes, $F_{t}$ is the value of fluorescence after $t$ minutes of incubation with OMVs and $F_{\max }(100 \%$ fluorescence) is the value of fluorescence after addition of Triton $\times 100$ to disperse maximally the probes.

\section{RESULTS}

\subsection{Characterization of OMVs Produced by E. coli WAM 1824}

Recently, Balsalobre et al. demonstrated that physiologically active HlyA is associated with OMVs produced by laboratory strains and also from natural and clinical $E$. coli isolates. However, as the amount of OMVs and the percentage of the soluble and OMVs associated HlyA were likely to vary markedly depending on the strain [8], we characterized OMVs obtained from E. coli WAM 1824 strain.

OMVs were purified from the culture filtrate of $E$. coli WAM 1824. Many bilayered spherical vesicles with diameters ranging from 50 to $200 \mathrm{~nm}$ were observed by electron microscopy (data not shown). The lipid composition of these vesicles was analyzed by thin layer chromatography, showing that the two predominant lipid species are phosphatidylethanolamine (PE) and cardiolipin (CL), demonstrating that these vesicles arise indeed from the bacterial outer membrane (data not shown).

The presence of HlyA in OMVs produced by E. coli WAM 1824 is shown in Figure 1(a). This figure shows the SDS-PAGE polypeptide profile of OMVs. A band with an electrophoretic mobility corresponding to HlyA (MW $110 \mathrm{kDa}$ ) was observed whose identity was confirmed by Western blot analysis employing polyclonal antibodies directed against purified HlyA (Figure 1(b)). The others bands that appear in SDS-PAGE stained by Comassie stain, correspond to periplasmic and outer membrane proteins also present in OMVs. 


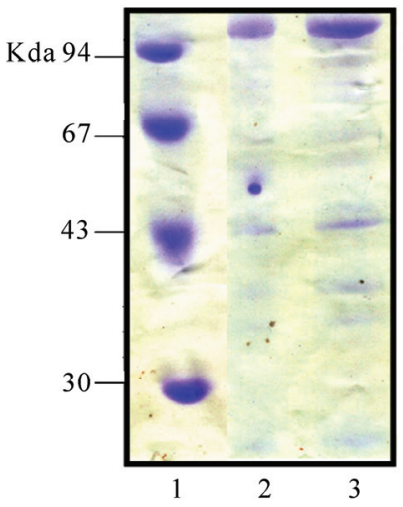

(a)

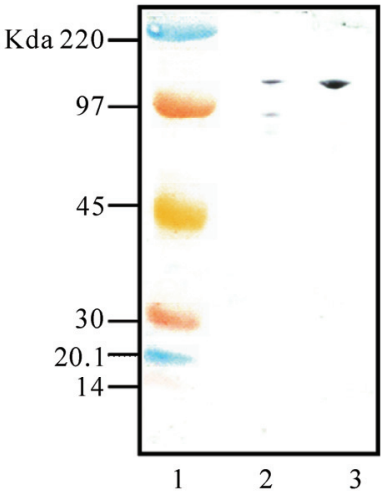

(b)
Figure 1. HlyA is present in WAM 1824 outer membrane vesicles. (a) SDS-PAGE 10\%, stained by Coomasie. 1: LMW, 2: WAM 1824 OMVs (10 $\mu \mathrm{g}$ prot), 3: WAM 783 OMVs $(10 \mu \mathrm{g})$; (b) Western-Blot, using anti-HlyA polyclonal antibodies. 1: Rainbow molecular weight, 2: OMV's (100 ng prot), 3: Standard HlyA (50 ng).

\subsection{Comparison of Hemolytic Activity between Free-HLYA and OMVs Associated-HlyA}

The following experiments were done so as to compare the hemolytic activity between the OMVs associatedHlyA and free toxin, which was quantified by serial dilution assays against horse erythrocytes. The hemolytic activity was 40960 and $2560 \mathrm{HU} / \mathrm{ml}$ for OMVs associatedHlyA and free toxin, respectively. If we consider that total protein concentration in the OMVs is $2.7 \mathrm{mg} / \mathrm{mL}$ and $70 \%$ corresponds to HlyA (estimated from the intensity of the $110 \mathrm{kDa}$ band in a $10 \%$ SDS-PAGE gel, analyzed by Kodak digital Science 1D Software), so the approximate concentration of OMVs associated- HlyA is $1.710^{-5} \mathrm{M}$. These values allow us to calculate the specific activity which is $2.3710^{12} \mathrm{HU} / \mathrm{mol}$ and $2.0410^{11}$ $\mathrm{HU} / \mathrm{mol}$ for OMVs associated-HlyA and free-HlyA, respectively.

In order to confirm that the hemolytic activity of OMVs is solely due to HlyA and not to other proteins present in the OMVs, E. coli WAM 783 (strain with a deletion of $h l y C$ gen that encodes $\mathrm{HlyC}$, the protein responsible for HlyA acylation) was used as a source of OMVs. The presence of a protein corresponding to $110 \mathrm{kDa}$ in $10 \%$ SDS-PAGE confirms that inactive unacylated toxin is also associated to OMVs (Figure 1, lane 3). OMVs obtained from this strain do not have any hemolytic activity confirming that this activity in OMVs is a particular property due largely or totally to the presence of HlyA.

\subsection{Lipid Mixing Assays}

In order to determine whether the hemolytic action mechanism of OMVs associated-HlyA implies membrane fusion, we analyzed the lipid mixing between OMVs and ghost erythrocytes labeled with NBD-PE (donor) and $\mathrm{Rh}-\mathrm{PE}$ (acceptor). The ghost erythrocytes concentration used in lipid mixing assays is equal to the erythrocyte suspension used in hemolytic experiments.

$6.9 \%$ FRET decrease was detected between OMVs and labeled erythrocytes promoted by OMVs associatedHlyA. When free-HlyA was added to labeled erythrocytes, 8.1\% FRET decrease was obtained (Figure 2), which cannot be assigned to lipid mixing because unlabeled erythrocytes were absent in this experiment, so it should be attributed to protein insertion into lipid bilayer, increasing the distance between donor and acceptor [36].

The decrease in energy transfer in both experiments was similar, indicating that fusion events are not involved in the action mechanism of this toxin when it is OMVs associated; instead, a transfer process from OMVs to the target membrane must be involved. The presence of a high affinity specific receptor is not strictly necessary, as the same experiments were repeated with labeled large unilamellar vesicles (LUVs), giving similar FRET decrease (data not shown).

\subsection{HlyA Transfer from OMVs to Erythrocytes}

To study HlyA transfer from OMVs to erythrocytes, a serial diluted hemolytic assay was carried out as described previously in Experimental Procedures. Samples were centrifuged at $10,000 \mathrm{rpm}$ to separate ghost erythrocytes from OMVs. The pellets containing the ghosts were resuspended in SDS sample buffer, and electrophoresed in a $10 \%$ SDS-PAGE. Finally this gel was trans-

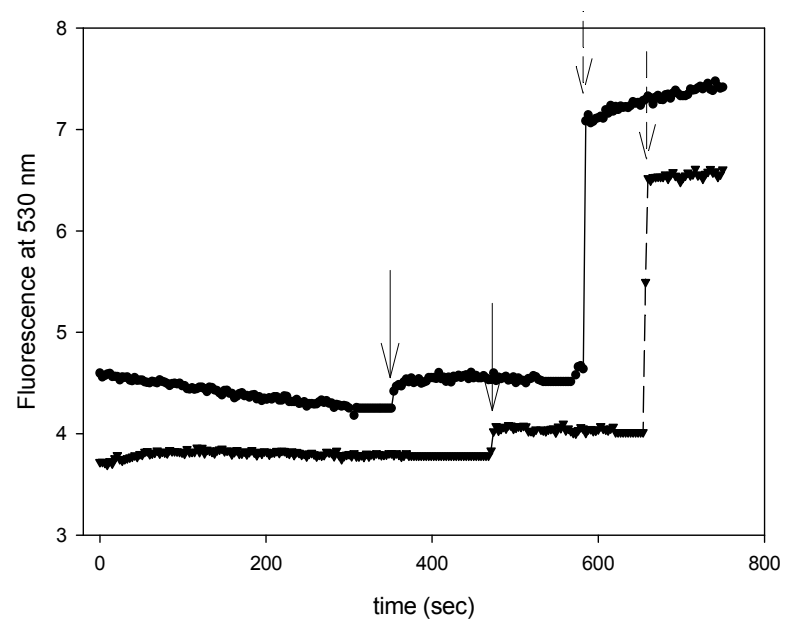

Figure 2. OMVs associated-HlyA does not induce membrane fusion. Lipid mixing assays between labeled erythrocytes with Rh-PE/NBD-PE and OMVs $(\bullet)$ or free-HlyA $(\boldsymbol{\nabla})$. Solid arrows correspond to the addition of OMVs or free-HlyA. Dotted arrows correspond to the addition of $0.2 \%$ Triton X-100 $\left(\mathrm{F}_{\max }\right)$. Total lipid concentration was $0.1 \mathrm{mM}$. $40 \mathrm{HU}$ for free-HlyA and OMVs associated-HlyA were used. 
ferred to nitrocellulose and HlyA was detected using a polyclonal rabbit anti-HlyA antibody. Figure 3(a) shows that HlyA is effectively transferred to erythrocytes. The amount of HlyA decreases with the decrease of the OMVs concentration, according to the decrease in the hemolytic activity observed (data not shown).

Another fact that confirms that fusion events are not involved in the hemolytic process mediated by OMVs is the absence of LPS in the ghost erythrocytes membranes after their incubation with OMVs. The detection of LPS was analyzed by a specific silver stain for LPS (BioRad silver stain kit, catalog 161-0445) of a 16\% SDS-PAGE. A typical LPS pattern is not observed in this sample (Figure 3(b), lane 1), instead some bands of glycoproteins of erythrocytes are observed.

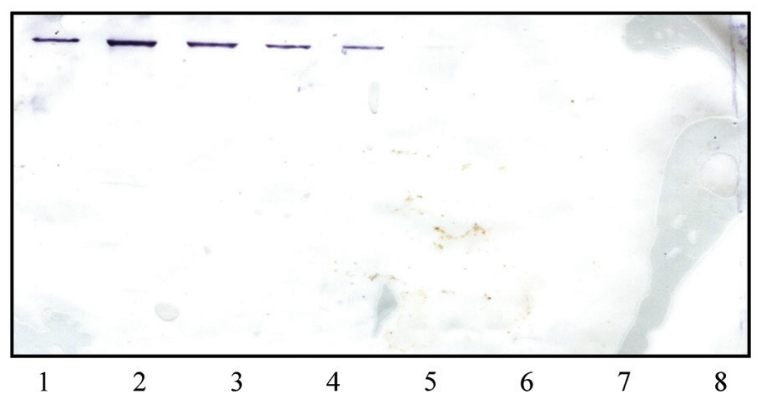

(a)

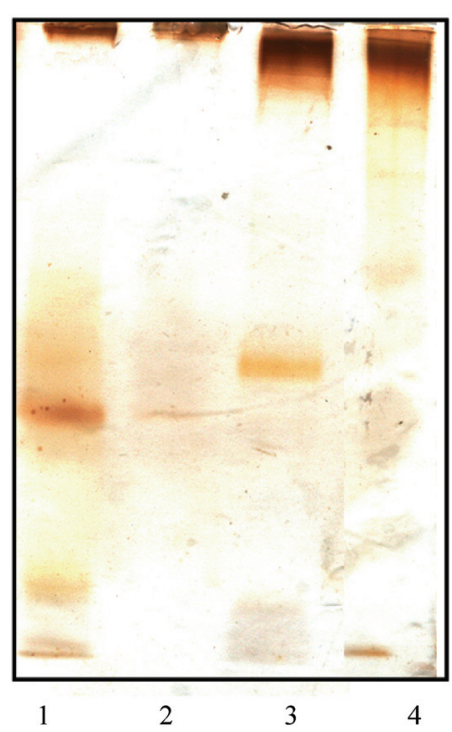

(b)

Figure 3. HlyA is transfered from OMVs to erythrocytes. (a) HlyA transference from OMVs to erythrocytes analyzed by western blot: lane 1: standard HlyA, 2-7: samples of ghosts erythrocytes previously incubated at $37^{\circ} \mathrm{C}$ during $30 \mathrm{~min}$ with serially diluted OMVs, 8: ghost erythrocytes; (b) Analysis of LPS content in ghost erythrocytes: lane 1: ghosts obtained from the hemolysis assay with OMVs, 2: ghosts, 3: OMVs and 4: Standard LPS from E. coli 011:B4.

\subsection{Hemolysis Kinetics}

Typical hemolysis time courses are shown in Figure 4. For both, OMVs associated-HlyA and free-HlyA, the time to reach $50 \%$ of hemolysis increases as the toxin concentration decreases. The hemolytic curves exhibit a define lag period for both free-HlyA and OMVs associatedHlyA. This lag period lengthens noticeably at decreasing concentrations of OMVs associated-HlyA, but it remains practically constant for free-HlyA as seen in Figure 5(a), indicating that the rate limiting step in the hemolysis process induced by OMVs associated-HlyA is the diffusion of vesicles in the aqueous medium, due to their higher molecular weight and volume compared to free-HlyA.

Initial rate of hemolysis obtained from the linear portion of the kinetic curve are shown in Figure 5(b). Vir-

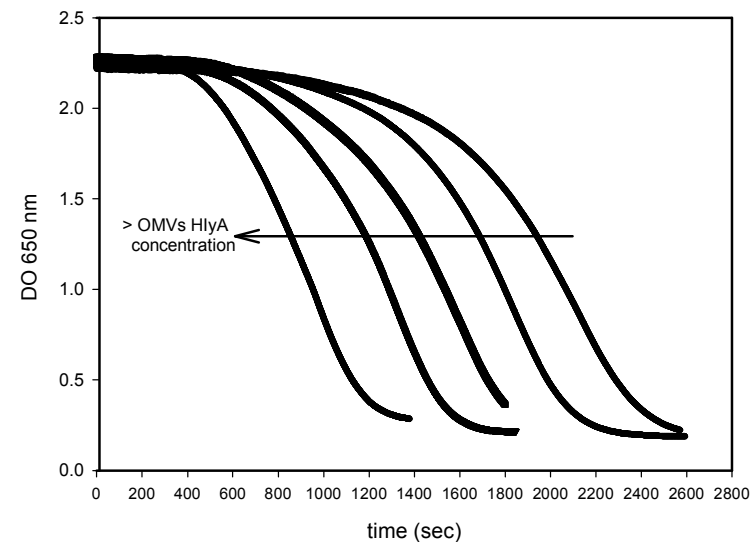

(a)

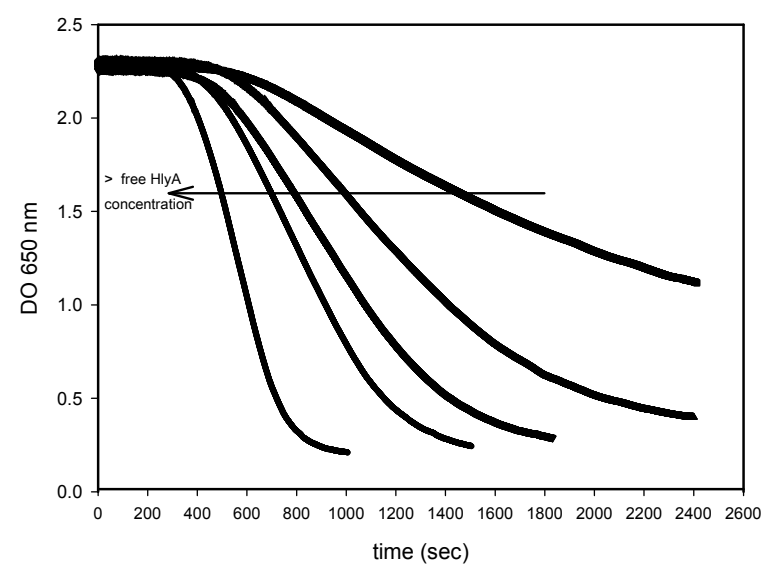

(b)

Figure 4. Kinetics of hemolysis induced by OMVs associated-HlyA (a) and free-HlyA (b). The real time kinetic assays for hemolysis were performed in the following manner: $500 \mu 1$ of standardized erythrocytes and $500 \mu \mathrm{l}$ of hemolysis buffer containing $10 \mathrm{mM} \mathrm{CaCl}_{2}$ were placed into a cuvette. Hemolysis started when the toxin was injected into the cuvette, and the absorbance at $650 \mathrm{~nm}$ was measured. The arrow indicates toxin concentration increase. 


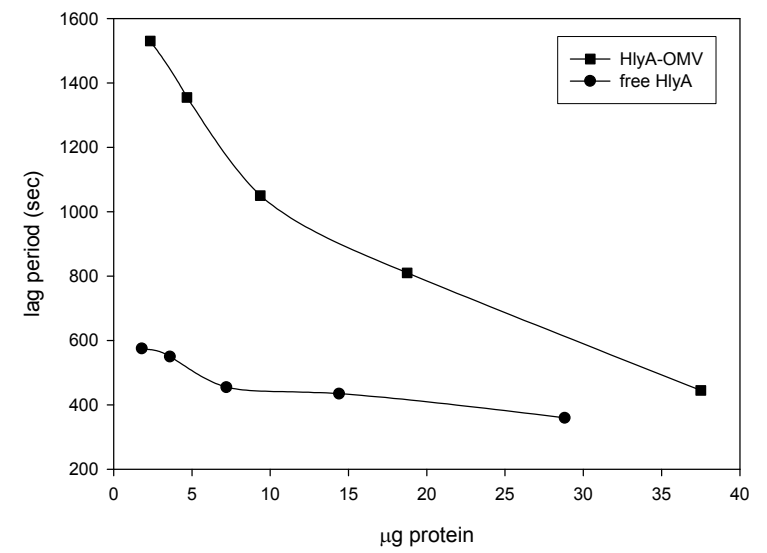

(a)

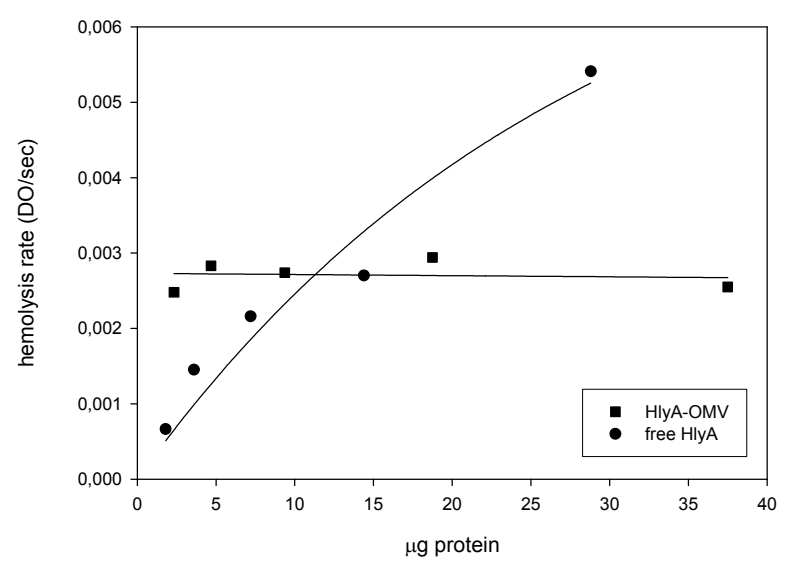

(b)

Figure 5. Initial hemolytic rate (a) and lag time (b) as a function of OMVs associated-HlyA $(\bullet)$ and free-HlyA ( $(\boldsymbol{)})$ concentration. Initial rates (linear portion) and lag time were obtained from Figure 5 at different toxin concentrations.

tually constant values were obtained with OMVs associated-HlyA for all the concentrations studied, while a decrease in initial rates as concentrations decrease was observed for free-HlyA.

\subsection{Erythrocytes Specificities of Free-HLYA and OMVs Associated-HlyA}

To characterize the hemolytic efficiency of OMVs associated-HlyA on different species of mammalian cells, we chose rabbit and horse erythrocytes, which were the most commonly used and characterized in previous studies related with the hemolytic action mechanism of this toxin. In Figures 6(a) and (b) it can be seen that in both cases, rabbit erythrocytes are more sensitive than those of horse, as demonstrated in earlier results on the characterization of the lytic action of HlyA [37]. The $\mathrm{D}_{50}$ values (amount of toxin that produces $50 \%$ of hemolysis) are 0.46 and $2.81 \mathrm{nM}$ for OMVs associated-HlyA for rabbit and horse erythrocytes respectively.
The dose-response curves indicate a higher lytic efficiency of OMVs associated-HlyA (Figure 6(a)) as compared to free-HlyA (Figure 6(b)), shown by the $\mathrm{D}_{50}$ on horse erythrocytes of 2.81 and $22.5 \mathrm{nM}$, respectively. Moreover, analysis of curves of hemolytic activity against concentration using SigmaPlot (Jandel Scientific, San Rafael, CA) fitted a sigmoidal curve in both cases. In the case of horse cells, data fit a sigmoid 4 parameter curve, while the rabbit ones fit a logistic 3 parameter curve, demonstrating a different cooperativity in the hemolysis process. The same behavior is observed for OMVs associated-HlyA as for free-HlyA.

\section{DISCUSSIONS}

Recently, it was demonstrated the presence of active HlyA associated with OMVs produced by natural and clinical E. coli isolates. Despite a great deal of published

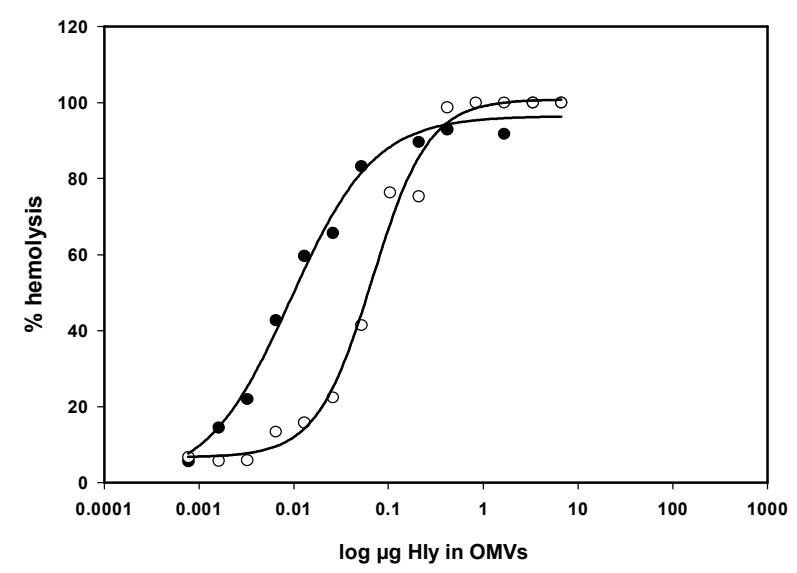

(a)

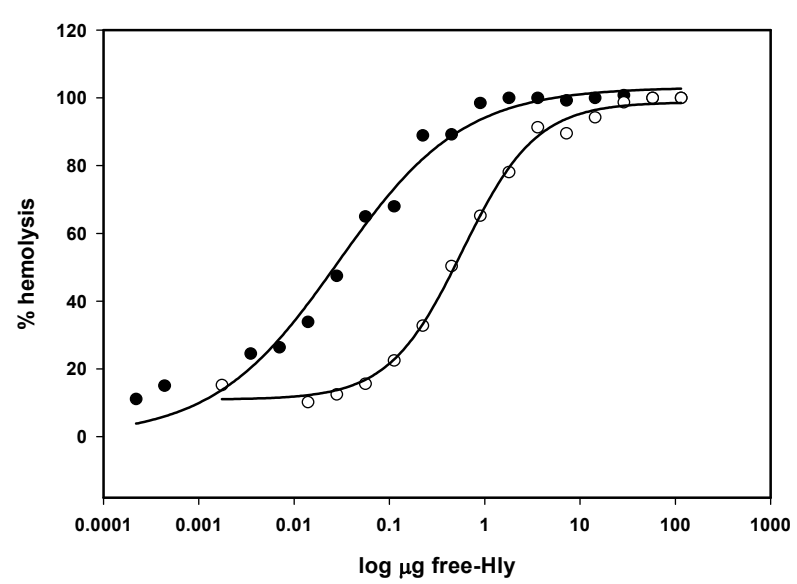

(b)

Figure 6. Hemolysis induced by OMVs associated-HlyA and free-HlyA on erythrocytes of different species. Hemolytic activity of OMVs associated-HlyA (a) and free-HlyA (b), tested with rabbit erythrocytes $(\bullet)$ and horse erythrocytes $(\mathrm{O})$. 
data regarding the activity of soluble HlyA secreted by $E$. coli, nothing was known regarding the action mechanism of OMVs associated HlyA. First, we demonstrated in this research, the presence of HlyA associated to OMVs produced by a laboratory $E$. coli strains (WAM 1824). We demonstrated the presence of HlyA associated to these vesicles as seen in SDS-PAGE polypeptide profile and Western blot analysis employing polyclonal antibodies directed against purified HlyA (Figure 1). The toxin associated to OMVs has a specific activity one order of magnitude higher than the free-HlyA. It is important to note that this protein is the only one responsable of the hemolytic activity as OMVs produced by strain WAM 783 (strain encoding the inactive form of HlyA, ProHlyA) are not hemolytic.

In order to determine whether the hemolytic action mechanism of OMVs associated-HlyA implies membrane fusion, we analyzed the lipid mixing between OMVs and labeled ghost erythrocytes. The small FRET decrease obtained could be attributed to protein insertion into lipid bilayer, as similar values were obtained in the presence of free-HlyA (Figure 2). These values are similar to those obtained in the study of HlyA insertion area using also FRET experiments with labeled membranes [36]. As for free-HlyA [38], fusion events are not involved in the action mechanism of the toxin associated with OMVs; instead, a transfer process from OMVs to the target membrane must be involved. The transference of HlyA as a mechanism was confirmed by the presence of HlyA and by the absence of LPS in the pellet obtained after incubation of ghost erythrocytes with OMVs (Figure 3). These results effectively demonstrate that the hemolysis is caused by the transference of HlyA and not OMVs-cell membranes fusion.

Although fusion events have been described for leukotoxin from $A$. actinomycetemcomitans, another RTX toxin, this could be specific for this RTX toxin [39], as the secretion of leukotoxin differs from that of other RTX proteins in that it remains associated with intact cells even in the presence of functional ltxBD genes [40].

The common mechanism of the lytic action of HlyA implies the diffusion of a soluble secreted toxin from bacteria, through aqueous medium, to reach the target cells. Now, our results demonstrate that a large number of toxin molecules are concentrated and transported by OMVs and then transfer to target cells.

The different behavior in the kinetics of hemolysis observed in Figure 4 for OMVs associated-HlyA and free-HlyA can be explained considering that, in the first case, the limiting step is the slow diffusion of OMVs in the aqueous medium that increases the lag time. Once OMVs reach the erythrocyte, a large number of toxin molecules which are concentrated on OMVs, are all together transferred to target cells either as a monomer or as a preassembled oligomer. On the contrary, the limiting step for free-HlyA is the number of toxin molecules that reach the target cells, due to the fact that they decrease as a function of toxin concentration. This can explain the high specific hemolytic activity for OMVs-associated HlyA in comparison with the hemolytic activity of freeHlyA. The fact that HlyA can be transfer in an oligomeric structure is possible because we have recently demonstrated that an oligomer is necessary for the occurrence of hemolysis [41]. What's more, the adoption of a cytolytically active oligomeric conformation in OMVs was described for enterobacterial ClyA cytotoxin [18].

The transference is direct from the OMVs to the erythrocytes without the delivery of the toxin to the medium. This is demonstrated by the differences found in the hemolysis kinetic studies (Figure 4). This conclusion is also supported by results published by Balsalobre et al., who demonstrated that HlyA is tightly associated to vesicles appearing in a soluble form only after the membrane structure of the OMVs is disrupted by detergents [8]. This transfer process does not need the presence of a specific high affinity receptor because the same effect was observed when LUVs labeled with Rh-PE and NBD-PE were used (data not shown).

Rabbit and horse erythrocytes, were the most commonly erythrocytes used and characterized in previous studies related with the hemolytic action mechanism of this toxin. In Figures 6(a) and (b) it can be seen that in both cases, rabbit erythrocytes are more sensitive than those of horse, as demonstrated in earlier results on the characterization of the lytic action of HlyA [37]. For rabbit erythrocytes a smoother hemolytic curve was obtained in comparison to that of horse erythrocytes. This fact may be due to the presence of high proportion of high affinity receptors in the horse erythrocytes in comparison to those of rabbit. The higher lytic efficiency of the toxin with rabbit cells could be due to the presence of a large number of low affinity binding sites i.e. membrane phospholipids, which facilitate the concentration of the toxin in the membrane (Herlax et al., unpublished results).

Currently, the presence of a receptor is a contradictory point in the elucidation of the action mechanism of this toxin, probably due that experiments were done with erythrocytes from different mammalian species. Glycophorin was described as a receptor in horse and human erythrocytes [42], while another group proposed that the hemolytic process does not depend on the receptor presence [43]. Anyway, the same behavior for HlyA against to erythrocytes of different species is observed for OMVs associated-HlyA as for free-HlyA.

Finally, it is important to remember that our previous results support the hypothesis that HlyA is secreted to the external medium as a LPS-HlyA complex, in which the main action of LPS is to maintain the protein stability in solution, while it only indirectly affects HlyA lytic 
activity [44]. The presence of LPS in the toxin sample used in those studies, which was obtained by precipitation techniques from a culture supernatant without the ultracentrifugation step, could be due to some OMVs contamination. This is also supported by the presence of phospholipid in toxin samples purified by methods such as ammonium sulphate precipitation and exclusion chromatography [45]. Due to the fact that OMVs associatedHlyA represents a very high percentage of toxins in the culture supernatants, results on biological effects of HlyA published up to date, mainly those in which sublytic concentrations were used, should be revised as small amounts of LPS present in the OMVs can stimulate the release of inflammatory mediators [46].

In conclusion, our results demonstrate that OMVs constitute an alternative secretion mechanism for HlyA that result in the toxin reaching higher concentrations without altering its lytic action mechanism.

The observation that certain virulence factors are enrichhed in vesicles suggests that OMVs may have a key role in bacterial pathogenesis by mediating transmission of active virulence factors and other bacterial envelopment component to host cells. Numerous OMVs associated virulence factors have been shown to induce cytotoxicity confer vesicle binding to and invasion to host cells and modulate the immune response. On the other hand, similar to bacterial-host cell interactions, OMVscell interactions can be altered by manipulating the expression of outer membrane proteins in bacteria. The manipulation of OMVs adherence and the possibility to create chimeras between the N-terminal half of HlyA, which contains the pore forming domain, and binding domains to specific proteins in the surface of a cell of interest (e.g. cancerous cells) should be useful for numerous applications, redirecting this engineering OMVs to specific cell types in order to achieve a desired therapeutic response.

\section{ACKNOWLEDGEMENTS}

This work was partially supported by grants from UNLP, CICPBA and ANPCyT-Argentina. LSB is a member of the "Carrera del Investigador", CICPBA, Argentina; VH is a member of the "Carrera del Investigador", Consejo Nacional de Investigaciones Científicas y Tecnológicas (CONICET), Argentina and MFH is a Fellow from CONICET, Argentina AMB is a member the "Carrera del Técnico y Profesional de Apoyo", CONICET.

\section{REFERENCES}

[1] Coote, J.G. (1992) Structural and fuctional relationships among the RTX toxin determinants of gram-negative bacteria. FEMS Microbiology Reviews, 88, 137-162.

[2] Cavalieri, S., Bohach, G. and Synder, I. (1984) Escherichia coli a-hemolysin characteristics and probable role in pathogenicity. Microbiology Reviews, 48, 326-343.

[3] Welch, R.A. (1991) Pore-forming cytolysins of gram-nega- tive bacteria. Molecular Microbiology, 5(3), 521-528.

[4] Ludwig, A. and Goebel, W. (1999) The family of the multigenic encoded RTX toxin. In: Alouf, J.E. and Freer, J.H., Eds., The Comprehensive Source-Book of Bacterial Protein Toxins, Academic Press, 330-348.

[5] Stanley, P., Korornakis, V. and Hughes, C. (1998) Acylation of Escherichia coli hemolysin: A unique protein lipidation mechanism underlying toxin fuction. Microbiology and Molecular Biology Reviews, 62(2), 309-333.

[6] Jarchau, T., Chakraborty, T., Garcia, F. and Goebel, W. (1994) Selection for transport competence of C-terminal polypeptides derived from Escherichia coli hemolysin: The shortest peptide capable of autonomous HlyB/HlyDdependent secretion comprises the C-terminal 62 amino acids of HlyA. Molecular and General Genetics, 245, 53-60.

[7] Koronakis, V., Koronakis, E. and Hughes, C. (1989) Isolation and analysis of the $\mathrm{C}$-terminal signal directing export of Escherichia coli hemolysin protein across both bacterial membranes. European Molecular Biology Organization Journal, 8(2), 595-605.

[8] Balsalobre, C., Silvan, J.M., Berglund, S., Mizunoe, Y., Uhlin, B.E. and Wai, S.N. (2006) Release of the type I secreted alpha-haemolysin via outer membrane vesicles from Escherichia coli. Molecular Microbiology, 59(1), 99-112.

[9] McBroom, A.J., Johnson, A.P., Vemulapalli, S. and Kuehn, M.J. (2006) Outer membrane vesicle production by Escherichia coli is independent of membrane instability. The Journal of Bacteriology, 188(15), 5385-5392.

[10] McBroom, A.J. and Kuehn, M.J. (2007) Release of outer membrane vesicles by Gram-negative bacteria is a novel envelope stress response. Molecular Microbiology, 63(2), 545-558.

[11] Beveridge, T.J. (1999) Structures of Gram-negative cell walls and their derived membrane vesicles. The Journal of Bacteriology, 181(16), 4725-4733.

[12] Horstman, A.L. and Kuehn, M.J. (2000) Enterotoxigenic E. coli secretes active heat-labile enterotoxin via outer membrane vesicles. Journal of Biological Chemistry, 275(17), 12489-12496.

[13] Devoe, I.W. and Gilchrist, J.E. (1973) Release of endotoxin in the form of cell wall blebs during in vitro growth of Neisseria meningitis. Journal of Experimental Medicine, 138, 1156-1167.

[14] Keenan, J., Day, T., Neal, S., Cook, B., Perez-Perez, G., Allardyce, R. and Bagshaw, P. (2000) A role for the bacterial outer membrane in the pathogenesis of Helicobacter pylori infection. FEMS Microbiology Letters, 182(2), 259-264.

[15] Fiocca, R., Necchi, V., Sommi, P., Ricci, V., Telford, J. Cover, T.L. and Solcia, E. (1999) Release of Helicobacter pylori vacuolating cytotoxin by both a specific secretion pathway and budding of outer membrane vesicles. Uptake of released toxin and vesicles by gastric epithelium. Journal of Pathology, 188, 220-226.

[16] Kato, S., Kowashi, Y. and Demuth, D.R. (2002) Outer membrane-like vesicles secreted by Actinobacillus actionmycetemcomitans are enriched in leukotoxin. Microbial Pathogenes, 32, 1-13.

[17] Wai, S.N., Takade, A. and Amako, K. (1995) The release of outer membrane vesicles from the strains of enterotoxigenic Escherichia coli. Microbiology and Immunology, 39(7), 451-456.

[18] Wai, S.N., Lindmark, B., Soderblom, T., Takade, A., Weste- 
rmark, M., Oscarsson, J., Jass, J., Richter-Dahlfors,V., Mizunoe, Y. and Uhlin, B.E. (2003) Vesicle-mediated export and assembly of pore-forming oligomers of the enterobacterial ClyA cytotoxin. Cell, 115(1), 25-35.

[19] Chi, B., Qi, M. and Kuramitsu, H.K. (2003) Role of dentilisin in Treponema denticola epithelial cell layer penetration. Research in Microbiology, 154(9), 637-643.

[20] Kuehn, M. and Kesty, N. (2005) Bacterial outer membrane vesicles and the host-pathogen interaction. Genes and Development, 19(22), 2645-2655.

[21] Mashburn-Warren, L. and Whiteley, M. (2006) Special delivery: Vesicle trafficking in prokaryotes. Molecular Microbiology, 61(4), 839-846.

[22] McBroom, A. and Kuehn, M.J. (2005) Outer membrane vesicles. In: Curtiss, R. III, et al., Eds., EcoSal-Escherichia coli and Salmonella: Cellular and Molecular Biology, ASM Press, Washington, DC.

[23] Kolling, G.L. and Matthews, K.R. (1999) Export of virulence genes and shiga toxin by membrane vesicles of Escherichia coli O157:H7. Applied and Environmental Microbiology, 65(5), 1843-1848.

[24] Moayeri, M. and Welch, R. (1997) Prelytic and lytic conformation of erythrocyte-associated Escherichia coli hemolysin. Infection and Immunity, 65(6), 2233-2239.

[25] Boehm, D., Welch, R. and Snyder, I. (1990) Calcium is requiered for binding of Escherichia coli hemolysin (HlyA) to erythrocyte membrane. Infection and Immunity, 58(6), 1951-1958.

[26] Markwell, M.A., Haas, S.M., Bieber, L.L. and Tolbert, N.E. (1978) A modification of the lowry procedure to simplify protein determination in membrane and lipoprotein samples. Analytical Biochemistry, 87(1), 206-210.

[27] Karkhanis, Y.D., Zeltner, J.Y., Jackson, J.J. and Carlo, D.J. (1978) A new and improved microassay to determine 2keto-3-deoxyoctonate in lipopolisaccharide of Gram-negative bacteria. Annals of Clinical Biochemistry, 85, 595-601.

[28] Chen, P., Toribara, T. and Warner, H. (1956) Microdetermination of phosphorus. Annals of Chemistry, 28, 17561758.

[29] Laemmli, U.K. (1970) Cleavage of structural proteins during the assembly of the head of bacteriophage T4. Nature, 227(5259), 680-685.

[30] Fomsgaard, A., Freudenberg, M. and Galanos, C. (1990) Modification of the silver staining technique to detect lipopolysaccharide in polyacrylamide gels. Journal of Clinical Microbiology, 28(12), 2627-2631.

[31] Towbin, H., Staehelin, T. and Gordon, J. (1979) Electrophoretic transfer of proteins from polyacrylamide gels to nitrocellulose sheets: Procedure and some applications. Proceedings of the National Academy of Sciences, 76(9), 4350-4354.

[32] Snyder, I.S. and Zwadyk, P. (1969) Some factors affecting production and assay of Escherichia coli hemolysin. Journal of General Microbiology, 5, 133-143.

[33] Soloaga, A., Ramírez, J.M. and Goñi, F.M. (1998) Reversible denaturation, self-aggregation, and membrane activity of Escherichia coli alpha-hemolysin, a protein stable in $6 \mathrm{M}$ urea. Biochemistry, 37(18), 6387-6393.

[34] Mayer, L.D., Hope, M.J. and Cullis, P.R. (1986) Vesicles of variable sizes produced by a rapid extrusion procedure. Biochimica et Biophysica Acta, 858, 161-168.

[35] Struck, D.K., Hoekstra, D. and Pagano, R.E. (1981) Use of resonance energy transfer to monitor membrane fusion. Biochemistry, 20, 4093-4099.

[36] Herlax, et al, unpublished results.

[37] Rennie, R.P. and Arbuthnott, J.P. (1974) Partial characterization of Escherichia coli haemolysin. Journal of Medical Microbiology, 7(2), 179-188.

[38] Ostolaza, H., Bartolome, B., Ortiz de Zarate, I., de la Cruz, F. and Goñi, F.M. (1993) Release of lipid vesicle contents by the bacterial protein toxin alpha-haemolysin. Biochimica et Biophysica Acta, 1147, 81-88.

[39] Demuth, D.R., James, D., Kowash, Y. and Kato, S. (2003) Interaction of Actinobacillus actinomycetemcomitans outer membrane vesicles with HL60 cells does not require leukotoxin. Cellular Microbiology, 5(2), 111-121.

[40] Lally, E.T., Golub, E.E. and Kieba, I.R. (1991) Structure and function of the B and D genes of the Actinobacillus actionmycetemcomitans leukotoxin complex. Microbial Pathogenesis, 11, 111-121.

[41] Herlax, V., Maté, S., Rimoldi, O. and Bakás, L. (2009) Relevance of fatty acid covalently bound to Escherichia coli alpha-hemolysin and membrane microdomains in the oligomerization process. Journal of Biological Chemistry, 284, 25199-25210.

[42] Cortajarena, H., Goñi, F. and Ostolaza, H. (2001) Glycophorin as a receptor for Escherichia coli alphahemolysin in erythrocytes. Journal of Biological Chemistry, 276(16), 12513-12519.

[43] Valeva, A., Walev, I., Kemmer, H., Weis, S., Siegel, I., Boukhallouk, F., Wassenaar, T., Chavakis, T. and Bhakdi, S. (2005) Binding of Escherichia coli hemolysin and Activation of the target cells is not receptor-dependent. Journal of Biological Chemistry, 280(44), 36657-36663.

[44] Herlax, V., Tacconi de Alani, M. and Bakás, L. (2005) Role of lipopolysaccharide on the structure and function of alphahemolysin from Escherichia coli. Chemistry and Physics of Lipids, 135(2), 107-115.

[45] Ostolaza, H., Bartolome, B., Serra, J.L., Cruz, F. and Goñi, F.M. (1991) Alpha-haemolysin from E. coli. Purification and self-aggregation properties. Microbiology Letters, 280(2), 195-198.

[46] Czuprynski, C.J. and Welch, R.A. (1995) Biological effects of RTX toxins: The possible role of lipopolysaccharide. Trends in Microbiology, 3(12), 480-483. 